\title{
An Accelerated Metropolis Method
}

\author{
M. L. Stedman, W. M. C. Foulkes and M. Nekovee \\ The Blackett Laboratory, Imperial College, \\ Prince Consort Road, London SW7 2AZ, UK
}

(Dated: September 20, 2012)

\begin{abstract}
We propose a stochastic method to reduce the autocorrelation time of a general Monte Carlo method and apply it to the Variational Quantum Monte Carlo (VMC) simulation of full-core atoms. We achieve a reduction in autocorrelation time of at least a factor of four compared with the standard method. Further, we find an approximate analytic fit to our results which gives a comparable reduction in autocorrelation time at essentially no cost. Our analytic form is independent of the geometry of the system being modelled and, therefore, can be easily applied to the VMC simulation of solids; it may also prove useful in any MC simulation where there are widely varying length scales. Results are presented for C, F, and Si.
\end{abstract}




\section{INTRODUCTION}

Quantum Monte Carlo (QMC) methods provide a promising new way to tackle the manyparticle Schrödinger equation for atoms, molecules, and solids. QMC methods scale much better with the number of particles in the system than the established configuration interaction (CI) approach, yet can deliver comparable accuracy ${ }^{1}$. Pseudo-solids containing up to 1000 electrons have already been studied ${ }^{2}$, however, the widely varying length scales of full-core systems pose more of a challenge. The aim of the present work is to address the length scale problem in one particularly useful variant of QMC: Variational Quantum Monte Carlo.

Variational Monte Carlo (VMC) uses the Metropolis algorithm to evaluate the expectation values of various observables for a given many-particle trial wavefunction, $\Psi_{T}(\mathbf{R}), \mathbf{R}=$ $\left(\mathbf{r}_{1}, \mathbf{r}_{2}, \ldots, \mathbf{r}_{N}\right)$. The method proceeds by sampling the values of the observables at a series of positions, $\{\mathbf{R}\}$, in state space where a move from position $\mathbf{R} \rightarrow \mathbf{R}^{\prime}$ is proposed according to some stochastic transition matrix, $T\left(\mathbf{R} \rightarrow \mathbf{R}^{\prime}\right)$, and accepted with probability

$$
A\left(\mathbf{R} \rightarrow \mathbf{R}^{\prime}\right)=\min \left\{\frac{\left|\Psi_{T}\left(\mathbf{R}^{\prime}\right)\right|^{2}}{\left|\Psi_{T}(\mathbf{R})\right|^{2}} \frac{T\left(\mathbf{R}^{\prime} \rightarrow \mathbf{R}\right)}{T\left(\mathbf{R} \rightarrow \mathbf{R}^{\prime}\right)}, 1\right\}
$$

Provided the transitions proposed by $T$ are ergodic, the equilibrium distribution, $\left|\Psi_{T}(\mathbf{R})\right|^{2}$, will be sampled correctly.

The original formulation of the Metropolis algorithm ${ }^{3}$ made the generally applicable, but inefficient, choice that $T$ is symmetric. However, as has been shown by several authors ${ }^{4-8}$, considering both classical and quantum MC, the efficiency of a particular instantiation of the algorithm can be greatly enhanced by a suitable choice of $T\left(\mathbf{R} \rightarrow \mathbf{R}^{\prime}\right)$.

The drawback of the Metropolis method is that raw data samples of an observable are sequentially correlated. If it takes, on the average, $T_{C}$ MC moves before an observable is decorrelated (the autocorrelation time), then the effective number of independent observations in an MC run of length $N$ moves is only $N_{\text {eff }}=N / T_{C}$, a clear waste of computational resources.

In this paper we propose a general stochastic method (based on the well-known global optimisation method of simulated annealing ${ }^{9}$ ) to increase the computational efficiency of a MC method by reducing the autocorrelation time, and show it to be effective in the particular case of VMC simulation of full-core atoms. We also find an approximate analytic fit to our 
annealing results for VMC which gives a comparable increase in efficiency at essentially no cost.

\section{CHARACTERISING THE EFFICIENCY OF A METHOD}

Consider a MC run of $N$ moves split into $N_{B}$ blocks of length $L_{B} \gg T_{C}$ so that individual block averages may be considered independent. The variance of the mean energy for the run is then

$$
\sigma_{R}^{2}=\frac{\sigma_{B}^{2}}{N_{B}}=\frac{T_{C} \sigma^{2}}{N_{B} L_{B}}
$$

where $\sigma_{B}^{2}=N_{B}^{-1} \sum_{i=1}^{N_{B}}\left(E_{B, i}-\langle E\rangle\right)^{2}$ is the variance in the block averages; $\sigma^{2}=N^{-1} \sum_{i=1}^{N}\left(E_{i}-\right.$ $\langle E\rangle)^{2}$ is the variance in an individual sample; and $\langle E\rangle=N_{B}^{-1} \sum_{i=1}^{N_{B}} E_{B, i}=N^{-1} \sum_{i=1}^{N} E_{i}$ is the mean energy for the run. The autocorrelation time is then given by

$$
T_{C}=\lim _{L_{B} \rightarrow \infty} L_{B}\left(\frac{\sigma_{B}}{\sigma}\right)^{2} .
$$

We see from (2) that

$$
\sigma_{B}^{2}=\frac{T_{C} \sigma^{2}}{L_{B}}
$$

and hence it is convenient to define the inefficiency, $\eta$, to be

$$
\eta:=L_{B} \sigma_{B}^{2}=T_{C} \sigma^{2}
$$

Thus, if we keep $L_{B}$ constant and assume that $\sigma^{2}$ is also constant ${ }^{10}, \eta$ is proportional to the autocorrelation time and, therefore, is an equivalent measure of the inefficiency of a method. Further, minimising $\eta$ is analogous to minimising $T_{C}$.

\section{ALGORITHM}

The major inefficiency in the standard MC algorithm is that it takes no account of the local environment when proposing a move. This is particularly detrimental in systems with widely varying length scales such as full-core atoms and solids. In this case, the step size is fixed by the smallest length scale (that of the core electrons) and, hence, the chemically interesting valence states have an artificially long autocorrelation time. Clearly it would be desirable to use small steps in the core region (where the wavefunction changes most rapidly) 
while using large ones in the valence region. Our task, therefore, is to make the proposed step size a (calculable) function of the local environment.

In order to ensure that the evaluation of moves is fast, we choose to propose single electron moves with

$$
\mathbf{r}_{i} \rightarrow \mathbf{r}_{i}^{\prime}=\mathbf{r}_{i}+\alpha_{i} \hat{\mathbf{F}}_{i}+\xi
$$

where $\xi$ is a Gaussian random 3-dimensional vector with a mean of 0 and variance $\delta_{i}$ in each component; $\alpha_{i}$ is a drift constant; and $\hat{\mathbf{F}}_{i}$ is the unit vector in the direction of the 'force' on a particle at $\mathbf{r}_{i}$ (in quantum MC we choose $\mathbf{F}_{i} \propto \nabla_{i} \ln \left|\Psi_{T}\right|$, which is often called the 'quantum force' on an electron; in classical MC the usual choice is $\mathbf{F}_{i}=-\nabla V$, where $V$ is the potential). Thus, the transition probability for a move $\mathbf{r}_{i} \rightarrow \mathbf{r}_{i}^{\prime}$ is defined as

$$
T\left(\mathbf{r}_{i} \rightarrow \mathbf{r}_{i}^{\prime}\right)=\frac{1}{\left(2 \pi \delta_{i}^{2}\right)^{3 / 2}} \exp \left\{-\left[\mathbf{r}_{i}^{\prime}-\left(\mathbf{r}_{i}+\alpha_{i} \hat{\mathbf{F}}_{i}\right)\right]^{2} / 2 \delta_{i}^{2}\right\}
$$

where the parameters $\alpha_{i}$ and $\delta_{i}$ depend on the value of some yet to be specified function of position, $f\left(\mathbf{r}_{i}\right)$, describing the local environment. Stated mathematically, we seek the mapping

$$
\mathcal{M}: f\left(\mathbf{r}_{i}\right) \rightarrow\left(\alpha_{i}, \delta_{i}\right)
$$

which minimises the inefficiency. For classical MC, our form of $T\left(\mathbf{r}_{i} \rightarrow \mathbf{r}_{i}^{\prime}\right)$ is similar to those proposed by both Rao and Berne ${ }^{4}$ and Rossky et al. ${ }^{5}$. However, unlike previous schemes, we have not assumed that the displacement term increases as the magnitude of $\mathbf{F}_{i}$ increases. In fact, as we shall see later, $\mathbf{F}_{i}$ is large in the core of an atom (where we find that we need smaller than average displacements) and small outside. Another advantage of choosing $T$ to be a Gaussian is that we can, in principle, propose a move to any point in state space. Therefore, we never have to reject a move because the reverse move would be impossible.

Unfortunately, we have no a priori knowledge of the required mapping. Instead we apply the Metropolis method on itself by using the stochastic global optimisation method of simulated annealing to optimise a lookup table of $\left(\alpha_{i}, \delta_{i}\right)$ pairs keyed on $f\left(\mathbf{r}_{i}\right)$ such that the inefficiency, $\eta$, is minimised.

In the language of simulated annealing, $\eta$ is our 'cost function' while the 'control parameter' is determined empirically. The procedure can be stated as follows

1. Start with some initial lookup table and estimate the initial autocorrelation time, $T_{C}$, and the initial inefficiency, $\eta_{0}$. 
2. Set the block length $L_{B} \gg T_{C}$ so that averages over individual blocks can be regarded as independent random variables.

3. Modify the lookup table and run for many blocks to estimate the new inefficiency $\eta$.

4. Conditionally accept changes according to the annealing algorithm, reducing the 'temperature' slowly according to some 'cooling schedule'.

5. Iterate steps $3-5$ until convergence, periodically checking that the block length $L_{B} \gg$ $T_{C}$.

\section{RESULTS AND DISCUSSION}

We have applied this optimisation method to VMC simulations of several full-core atoms. In order to distinguish between results obtained with our new VMC method and the standard method given by, e.g., Fahy ${ }^{11}$, we shall term the new method 'directed VMC' (or simply $\mathrm{dVMC}$ ) in the rest of this paper.

\section{A. Trial wavefunctions}

We use variance-minimised ${ }^{12}$ trial wavefunctions of the Slater-Jastrow form

$$
\Psi_{T}(\mathbf{R})=\exp \left\{\sum_{(s, i)=(\uparrow, 1)}^{(\downarrow, N)} \chi\left(\mathbf{r}_{i}^{s}\right)-\sum_{(\uparrow, 1) \leq(s, i)<\left(s^{\prime}, j\right)}^{(\downarrow, N)} u_{s s^{\prime}}\left(\mathbf{r}_{i}, \mathbf{r}_{j}\right)\right\} D^{\uparrow} D^{\downarrow}
$$

The $D^{\uparrow \downarrow}$ represent Slater determinants of spin-up and spin-down single particle LDA orbitals; $\chi\left(\mathbf{r}_{i}\right)$ a parametrised one-body term; and $u_{s s^{\prime}}\left(\mathbf{r}_{i}, \mathbf{r}_{j}\right)$ a Jastrow function which correlates the motion of pairs of electrons. Following Fahy ${ }^{11}$, we define $u_{s s^{\prime}}\left(\mathbf{r}_{i}, \mathbf{r}_{j}\right)$ as

$$
u_{s s^{\prime}}\left(\mathbf{r}_{i}, \mathbf{r}_{j}\right)=\frac{A}{r_{i j}}\left\{1-\exp \left(-\frac{r_{i j}}{F_{s s^{\prime}}}\right)\right\}
$$

where $r_{i j}=\left|\mathbf{r}_{i}-\mathbf{r}_{j}\right|, A$ is a variational coefficient, and $F_{s s^{\prime}}$ is related to $A$ via the cusp conditions $^{13}$. We expand $\chi\left(\mathbf{r}_{i}\right)$ in Chebyshev polynomials, $T_{l}$, as suggested by Williamson et al. ${ }^{14}$,

$$
\chi\left(\mathbf{r}_{i}\right)= \begin{cases}B\left(\frac{L}{2}+r_{i}\right)\left(L-r_{i}\right)^{2}+r_{i}^{2}\left(L-r_{i}\right)^{2} \sum_{l=0}^{M} \alpha_{l} T_{l}(\bar{r}), & 0 \leq r_{i} \leq L \\ 0, & r_{i}>L\end{cases}
$$


where $B$ and $\left\{\alpha_{l}\right\}$ are variational coefficients, $L$ is chosen empirically, and $\bar{r}=\left(2 r_{i}-L\right) / L$ so that the range $(0, L)$ is mapped into the orthogonality interval of the Chebyshev polynomials, $(-1,1)$.

Trial wavefunctions of this form represent the state of the art for solid state QMC calculations, but other authors ${ }^{12,15}$ have used much more accurate trial functions for isolated atoms. Fortunately, small changes in the trial functions do not have much effect on the relative efficiencies of different VMC algorithms, so there is no need to use more sophisticated trial functions for this work.

\section{B. Defining the local description function}

The algorithm given in Sec. III is general. In order to completely define the dVMC method, we must specify the local description function, $f\left(\mathbf{r}_{i}\right)$, and its associated lookup table.

As we can see from Figure 1, a particularly good choice for atoms is the modulus of the quantum force at $\mathbf{r}_{i}$,

$$
\mathbf{F}_{i}(\mathbf{R})=\frac{1}{\sqrt{2}} \nabla_{i} \ln \left|\Psi_{T}(\mathbf{R})\right|
$$

which exhibits much underlying physical structure with clear core and valence regions ${ }^{16} \cdot\left|\mathbf{F}_{i}\right|$ is a better choice than $\left|\mathbf{r}_{i}\right|$ because it does not depend on a particular geometry. Thus, unlike Umrigar's spherical-polar method ${ }^{6}$, there is no need to keep track of the closest nucleus to an electron when proposing a move.

The large- and small-r limits of $\left|\mathbf{F}_{i}\right|$ can be understood by considering single-particle wavefunctions. If an electron is in a $1 s$ orbital very close to the nucleus, it feels an unscreened hydrogenic potential with nuclear charge $Z$, and the resulting hydrogenic wavefunction has $\left|\mathbf{F}_{i}\right|=Z / \sqrt{2}$. Conversely, in the large- $r$ limit where one electron is far outside the nucleus, it is probable that the remaining electrons are further in, and the electron sees an LDA wavefunction with the asymptotic form $e^{-|2 \epsilon|^{1 / 2} r}$; thus $\left|\mathbf{F}_{i}\right|$ reduces to $|\epsilon|^{1 / 2}$ where $\epsilon$ is the LDA eigenvalue for the outermost orbital.

Our lookup table of $\left(\alpha_{i}, \delta_{i}\right)$ pairs is keyed on logarithmic ranges of $f\left(\mathbf{r}_{i}\right)$. Specifically, we define table elements such that the $j$ th element, $j=1, \ldots, N$, covers the range $m^{j-1} f_{1} \leq$ $f\left(\mathbf{r}_{i}\right)<m^{j} f_{1}$, where $m$ and $f_{1}$ are constant for a given $f\left(\mathbf{r}_{i}\right)$. We also define two special elements to trap underflow, $f\left(\mathbf{r}_{i}\right)<f_{1}$, and overflow, $f\left(\mathbf{r}_{i}\right) \geq m^{N} f_{1}$, respectively. 


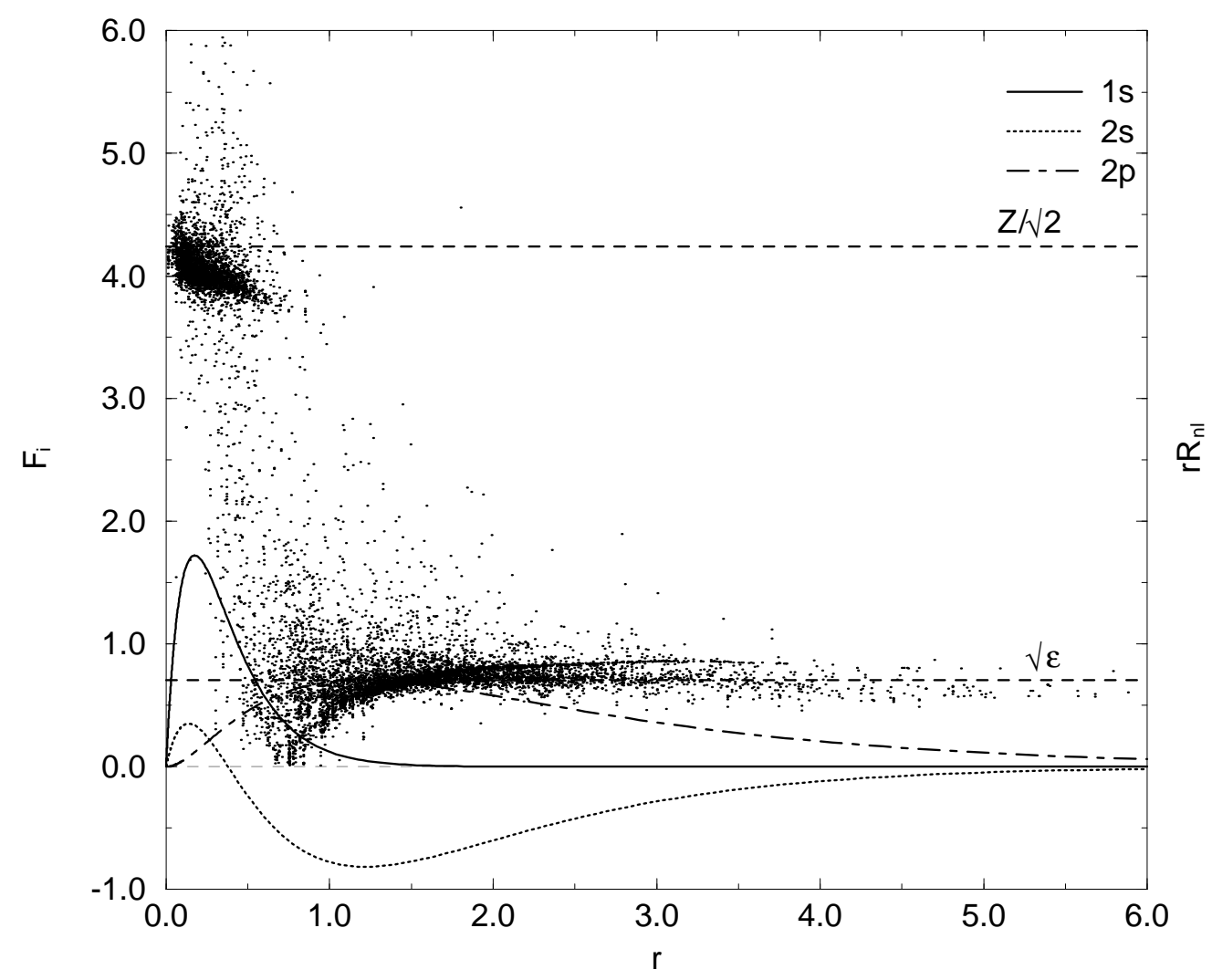

FIG. 1: Plot of $\left|\mathbf{F}_{i}\right|$ against $r$ for full-core Carbon (points) showing the large- and small- $r$ limits (dashed lines) and the radial one-particle LDA orbitals used in the Slater determiants, $r R_{n l} . \varepsilon$ is the modulus of the eigenvalue of the LDA $2 s$ orbital.

\section{An analytic form of $\mathcal{M}$}

Although robust, simulated annealing is woefully inefficient. If our directed method is to be practical, we must find an analytic form of the mapping

$$
\mathcal{M}_{\mathrm{VMC}}:\left|\mathbf{F}_{i}\right| \rightarrow\left(\alpha_{i}, \delta_{i}\right)
$$

Figure 2 shows the most significant elements in the lookup tables generated by simulated annealing for various full-core atoms. In each case, almost all of the samples of $\left|\mathbf{F}_{i}\right|$ fall within the range of one of two elements: a high $\left|\mathbf{F}_{i}\right|$ element corresponding to electrons in the core; and a low $\left|\mathbf{F}_{i}\right|$ element corresponding to electrons in the valence region. Both graphs show that, as expected, we require small moves in the core and larger ones in the valence region. 


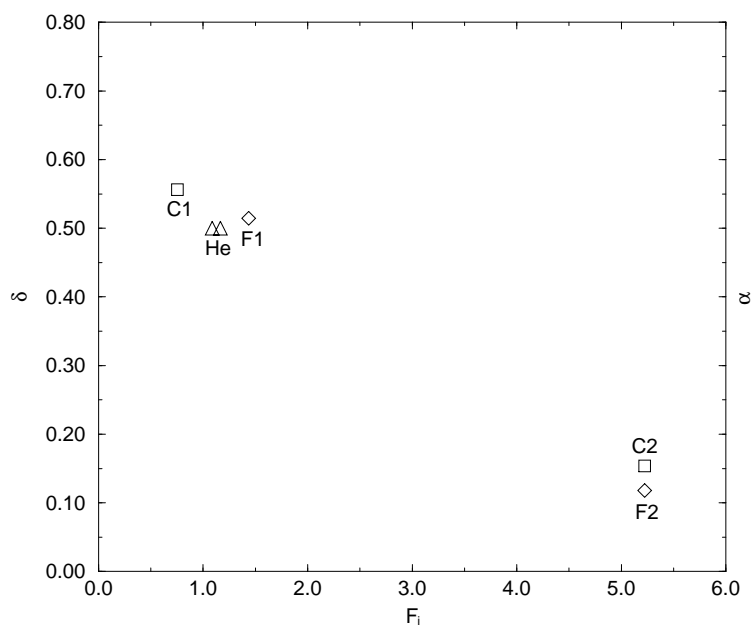

(a)

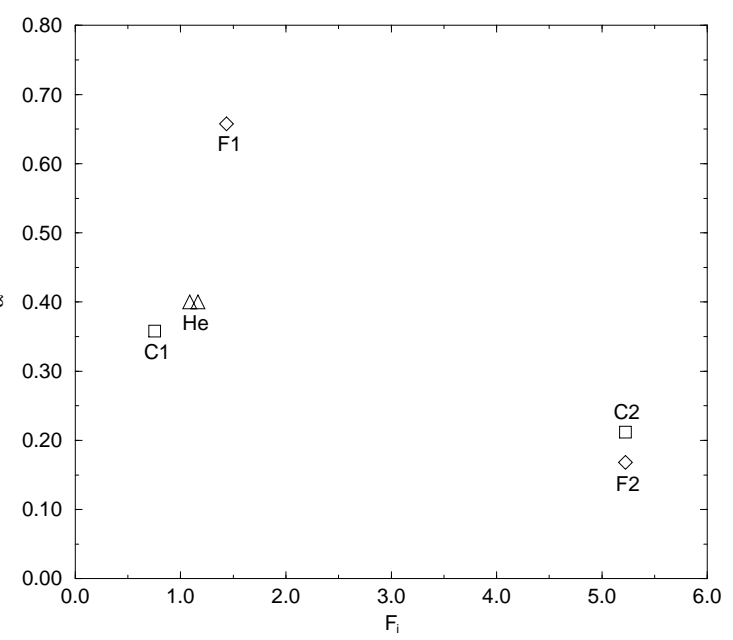

(b)

FIG. 2: (a) Step size, $\delta$, and (b) drift constant, $\alpha$, plotted against 'logarithmic' element mid-point, $m^{(j-1 / 2)} f_{1}$, for the most significant elements in the lookup tables generated by simulated annealing for full-core Carbon, Fluorine, and Helium atoms. We see that, as expected, optimal results are obtained by using small steps in the core and large steps in the valence region.

An approximate fit to the data (which has the right dimensions) is

$$
\delta_{i}=\frac{\kappa_{1}}{\left|\mathbf{F}_{i}\right|}, \quad \alpha_{i}=\frac{\kappa_{2}}{\left|\mathbf{F}_{i}\right|},
$$

where $\kappa_{1}$ and $\kappa_{2}$ are dimensionless constants to be determined empirically. In the results which follow, we term dVMC with moves proposed by these approximations dVMCF.

The $\left|\mathbf{F}_{i}\right|^{-1}$ form is appealing since, within a linear expansion about $\mathbf{R}$, it is proportional the distance to the nearest node, $d_{n}$, which can be reached by moving electron $\mathbf{r}_{i}$, and hence the rapidity of oscillation of the wavefunction. Thus, provided that $d_{n}$ is the only relevant length scale in the problem, the $\left|\mathbf{F}_{i}\right|^{-1}$ form follows naturally.

\section{Comparison of methods}

Table I shows the autocorrelation times of the dVMC algorithms together with those for the standard method and a form of the commonly-used ${ }^{4,6,7}$ Cartesian-coordinate directed method (CCdVMC). 
TABLE I: Autocorrelation times (measured in all-electron moves) of the algorithms described in the text for various full-core atoms. The uncertainty in $T_{C}$ is typically $10 \%$ of its value.

\begin{tabular}{|c|c|c|}
\hline Atom & Algorithm & $\eta \quad T_{C}$ \\
\hline \multirow[t]{4}{*}{$\mathrm{C}$} & VMC & 6.513 .3 \\
\hline & CCdvMC, $\Delta=1.1$ & $3.3 \quad 6.4$ \\
\hline & dVMC & $1.5 \quad 3.0$ \\
\hline & $\mathrm{dVMCF}, \kappa_{1}=\kappa_{2}=0.6$ & $1.4 \quad 2.8$ \\
\hline \multirow[t]{4}{*}{$\mathrm{F}$} & VMC & $25 . \quad 11.5$ \\
\hline & CCdVMC, $\Delta=0.7$ & $11.5 \quad 5.4$ \\
\hline & dVMC & $5.5 \quad 2.5$ \\
\hline & $\mathrm{dVMCF}, \kappa_{1}=0.75, \kappa_{2}=1.0$ & $\begin{array}{ll}5.5 & 2.5\end{array}$ \\
\hline \multirow[t]{3}{*}{$\mathrm{Si}$} & VMC & 97. 13. \\
\hline & CCdVMC, $\Delta=0.4$ & 41. \\
\hline & dVMCF, $\kappa_{1}=0.6, \kappa_{2}=0.7$ & 20. \\
\hline
\end{tabular}

The transition matrix for CCdVMC, $T_{C C}\left(\mathbf{r}_{i} \rightarrow \mathbf{r}_{i}^{\prime}\right.$ ), (derived from a linear expansion of $|\Psi|=e^{\ln |\Psi|}$ within a 3-dimensional box of side length $\Delta$ centered on $\mathbf{r}_{i}$ ) turns out to be

$$
T_{C C}\left(\mathbf{r}_{i} \rightarrow \mathbf{r}_{i}^{\prime}\right)=\left(\prod_{j=1}^{3} \frac{F_{i, j}}{\sqrt{2} \sinh \left(\Delta F_{i, j} / \sqrt{2}\right)}\right) \exp \left\{\sqrt{2} \mathbf{F}_{i} \cdot\left(\mathbf{r}_{i}^{\prime}-\mathbf{r}_{i}\right)\right\}
$$

inside the box $[-\Delta / 2, \Delta / 2]$, and 0 outside. Here $F_{i, j}$ is the $j$ th component of $\mathbf{F}_{i}$, and $\Delta$ is determined empirically.

We see that, despite its simplicity, dVMCF is as efficient as dVMC, and that both outperform CCdVMC. The main reason for this performance gain is that the structure of $\mathbf{F}_{i}$ causes CCdVMC to incorrectly propose large moves in the core region. Figure 3 shows that, unlike VMC and CCdVMC, the dVMC methods give high acceptance in both the physically significant core and valence regions. 


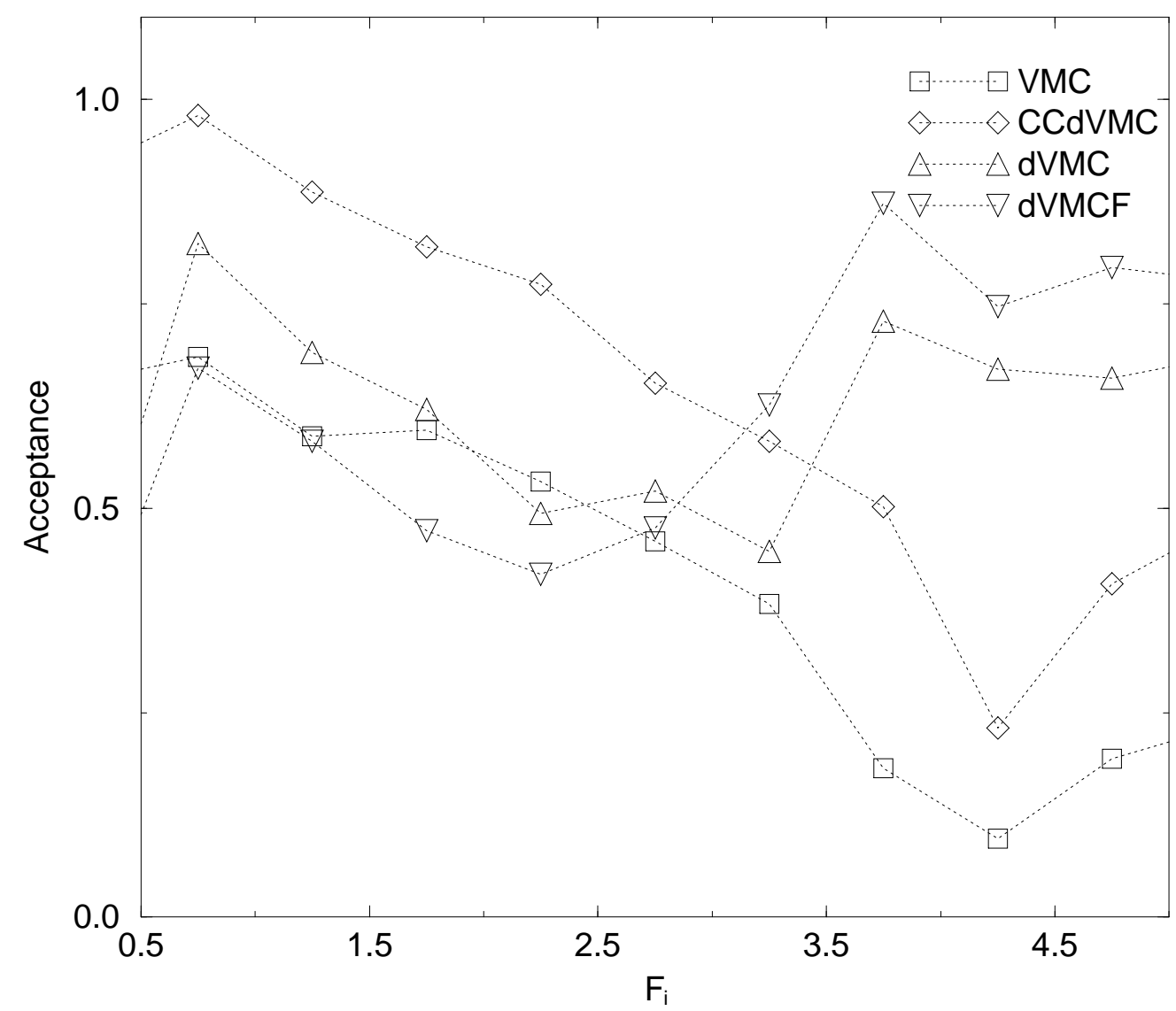

FIG. 3: Acceptance ratio against $\left|\mathbf{F}_{i}\right|$ for full-core Carbon showing that, unlike standard VMC and CCdVMC, both dVMC and dVMCF have high acceptance in the core region. When proposing moves with dVMCF, values of $\left|\mathbf{F}_{i}\right|<0.4$ were cut off to 0.4 .

\section{CONCLUSIONS}

We have shown that our general stochastic method works in the case of VMC simulation of full-core atoms, and found an approximate analytic fit to the annealing data which gives comparable results with minimal overhead. Although less efficient than Umrigar's spherical polar method ${ }^{6}$, our analytic form has the advantage of being more general in that it is independent of the geometry of the system being modelled. A quantity analogous to $\left|\mathbf{F}_{i}\right|$ exists in any VMC simulation (not just that for atoms, molecules, and solids), hence an $\left|\mathbf{F}_{i}\right|^{-1}$ dependence may prove useful wherever there are widely varying length scales.

Finally, we have demonstrated that the 'quantum force', F, has a lot of physical structure. In particular, the fact that $\left|\mathbf{F}_{i}\right|$ is large in the core means that directed vMC methods based on a linear expansion of $\left|\Psi_{T}(\mathbf{R})\right|$ around $\mathbf{R}$ are unlikely to be optimal for full-core systems. 


\section{ACKNOWLEDGMENTS}

We thank Dr. C. J. Umrigar for explaining his technique of coordinate-by-coordinate sampling in CCdVMC. We have also benefited from useful discussions with R. Gaudion.

1 R. N. Barnett, P. J. Reynolds, and W. A. Lester J., J. Phys. Chem. 91, 2004 (1987).

2 A. J. Williamson, G. Rajagopal, R. J. Needs, L. M. Fraser, W. M. C. Foulkes, Y. Wang and M. Y. Chou, Journal of the Korean Physical Society 29, S 116 (1996).

3 N. Metropolis, A. W. Rosenbluth, M. N. Rosenbluth, A. H. Teller, and E. Teller, J. Chem. Phys. 21, 1087 (1953).

4 M. Rao and B. J. Berne, J. Chem. Phys. 71(1), 129 (1979).

5 P. J. Rossky, J. D. Doll, and H. L. Friedman, J. Chem. Phys. 69(10), 4628 (1978).

${ }^{6}$ C. J. Umrigar, Phys. Rev. Lett. 71, 408 (1993).

7 Z. Sun, M. M. Soto, R. N. Barnett, and W. A. Lester J., J. Chem. Phys. 101(1), 394 (1994).

8 M. Mella, A. Lüchow, and J. Anderson, Chem. Phys. Lett. 265, 467 (1997).

9 E. Aarts and J. Korst, Simulated Annealing and Boltzmann Machines (Wiley, Chichester, 1989).

10 We note, as does Umrigar, that $\sigma^{2}$ depends on the system being sampled, but not on the VMC algorithm used.

11 S. Fahy, X. W. Wang, and S. G. Louie, Phys. Rev. B 42, 3503 (1990).

12 C. J. Umrigar, K. G. Wilson, and J. W. Wilkins, Phys. Rev. Lett. 60, 1719 (1988).

13 T. Kato, Communications on Pure and Applied Mathematics 10, 151 (1957).

14 A. J. Williamson, S. D. Kenny, G. Rajagopal, A. J. James, R. J. Needs, L. M. Fraser, W. M. C. Foulkes, and P. Maccallum, Phys. Rev. B 53, 9640 (1996).

15 K. E. Schmidt and J. W. Moskowitz, J. Chem. Phys. 93, 4172 (1990).

16 We use Hartree atomic units throughout: $\hbar=m=e=a_{0}=4 \pi \epsilon_{0}=1$. 